Article

\title{
Functionalization of Graphene Oxide with Low Molecular Weight Poly (Lactic Acid)
}

\author{
Mingwei Yuan ${ }^{1,2}$, Yike Chen ${ }^{3}$, Minglong Yuan ${ }^{3}$, Hongli Li ${ }^{3}$, Xiansong Xia ${ }^{1,2}$ and \\ Chengdong Xiong ${ }^{1, *}$ \\ 1 Chengdu Institute of Organic Chemistry, Chinese Academy of Sciences, Chengdu 610041, China; \\ yuanmw96@gmail.com (M.Y.); psxxs1982@gmail.com (X.X.) \\ 2 University of Chinese Academy of Sciences, Beijing 100049, China \\ 3 Engineering Research Center of Biopolymer Functional Materials of Yunnan, Yunnan Minzu University, \\ Kunming 650500, China; chenyike1992@gmail.com (Y.C.); yml@188.com (M.Y.); \\ hongli8302@gmail.com (H.L.) \\ * Correspondence: xiongcdcioc@gmail.com; Tel.: +86-28-8521-4764
}

Received: 27 December 2017; Accepted: 4 February 2018; Published: 12 February 2018

\begin{abstract}
In this paper, the hydroxyl groups on the surface of graphene oxide (GO) were used to initiate the ring-opening polymerization of a lactic acid $O$-carboxyanhydride. $\mathrm{GO}$ grafted with poly (L-lactic acid) molecular chains (GO-g-PLLA) was prepared. Lactic acid O-carboxyanhydride has a higher polymerization activity under mild polymerization conditions. Thus, the functionalization of the polymer chains and obtaining poly (lactic acid) (PLLA) was easily achieved by ring-opening polymerization with 4-dimethylaminopyridine (DMAP) as the catalyst. The results showed that with this method, PLLA can be rapidly grafted to the surface of GO in one step. As a result, the chemical structure of the GO surface was altered, improving its dispersion in organic solvents and in a PLLA matrix, as well as its bonding strength with the PLLA interface. We then prepared GO/PLLA and PLLA/GO-g-PLLA composite materials and investigated the differences in their interfacial properties and mechanical properties. GO-g-PLLA exhibited excellent dispersion in the PLLA matrix and formed excellent interfacial bonds with PLLA through mechanical interlocking, demonstrating a significant enhancement effect compared to PLLA. The water vapor and oxygen permeabilities of the GO-g-PLLA/PLLA composite decreased by $19 \%$ and $29 \%$, respectively.
\end{abstract}

Keywords: GO-g-PLLA; composite materials; water vapor; oxygen permeabilities

\section{Introduction}

Poly (lactic acid) (PLLA) is receiving considerable attention for conventional uses, such as a packaging material, for the production of agricultural film, and more recently, as composites for technical applications [1]. However, its wider application has been limited by its relatively slow crystallization rate, poor gas barrier performance, and poor flexibility [1]. To overcome these challenges, various methods (such as Copolymerization and blending) have been used to enhance the comprehensive performance of poly L-lactide (PLLA). The degree of crystallinity and mechanical properties can be changed by blending. Copolymerization can change the performance of many aspects, mainly based on the properties of the graft molecules. For example, a rigid molecule can improve the strength of the copolymer, while the flexible molecule can enhance the tensile properties of the copolymer [1,2]. Graphene and graphene oxide (GO) have been used in PLLA nanocomposites, and graphite or GO can increase the crystallinity and the heat resistant temperature of the nanocomposite [3-7]. In a study of graphene and GO-modified polylactic acid, the main method used was to directly blend graphene with polylactic acid. Feng et al. developed a versatile method by grafting polymers on $\mathrm{GO}$ to enhance the properties of nanocomposites [8,9]. Chaobin et al. reported 
a new type of PLLA-GO nanocomposite and showed that a stereocomplex crystal could be formed between PLLA and GO-g-PDLA. The incorporation of GO nanofillers lead to a lower crystallization activation energy of the stereocomplex and a higher crystallinity in solution casting samples [10]. The PLLA-GO nanocomposites were prepared by blending commercial PLLA with GO-g-PDLA, in which GO-g-PDLA was synthesized via ring-opening polymerization using modified GO as the initiator. According to the above literature, the crystallinity and heat resistance of PLLA can be significantly increased by modifying PLLA with graphene and GO.

Improving the gas barrier performance and flexibility of PLLA for the purpose of packaging and other uses is also necessary. Increasing the compatibility of nano polylactic acid composites and the adhesion force of the interface will improve their gas barrier property and their flexibility. Based on the "like dissolves like" principle, because inorganic nanoparticles are structurally different from polymers, modifying the structure of inorganic nanoparticles to improve their compatibility with polymers is essential. Albertsson et al. [11-14] used PLLA stereocomplex (SC) particles with a diameter of 300-500 nm to modify PLLA, and this initiated the interfacial crystallization of PLLA, resulting in a clear improvement in compatibility. In particular, the modification of PLLA with GO-PLLA SC composite particles significantly improved the thermal resistance and barrier properties of the material [12-14]. Based on the above analysis and the "like dissolves like" principle, this study aimed to prepare high barrier nanomaterial-PLLA membrane materials by modifying PLLA with structurally similar nanomaterials that were functionalized with low molecular weight PLLA. This study provides a new thought process and method to solve the key problem of nanomaterial-PLLA composite materials, and to lay a theoretical foundation for next-generation green food packaging materials.

\section{Experimental Methods}

\subsection{Materials}

\subsubsection{Preparation of Raw Materials}

Lactic acid O-carboxyanhydride (LacOCA) was prepared according to the literature [15-18]. The L-lactic aqueous solution was provided by Henan Jindan. Lactic Acid, LLC, lithium hydroxide ( $\mathrm{LiOH})$, ethanol, tert-butyl methyl ether, triphosgene, and tetrahydrofuran were obtained from the Chengdukelong chemical reagent PLLAnt, all with a purity of approximately $99 \mathrm{wt} \%$. GO was provided by Suzhou Carbon Abundance Graphene Science and Technology Co., Ltd., Suzhou, China, with a purity of $\sim 99 \mathrm{wt} \%$, a thickness of $0.6-1.0 \mathrm{~nm}$, a sheet diameter of $0.5-5 \mu \mathrm{m}$, with $1-2$ layers, and a specific surface area of $1000-1217 \mathrm{~m}^{2} / \mathrm{g}$. The PLLA was thermal film-grade PLLA from NatureWorks, LLC.

\subsubsection{Preparation of GO-g-PLLA}

Tetrahydrofuran (THF) $(150 \mathrm{~mL})$ and $0.067 \mathrm{~g}$ of GO (lactic acid O-carboxyanhydride of $0.5 / 100$ ) were added to a $250-\mathrm{mL}$ single-neck flask and sonicated for $1 \mathrm{~h}$ to evenly disperse the GO in the THF. LacOCA (14 g) was added to the above solution at room temperature and stirred until dissolved. After the solution became clear, $0.046 \mathrm{~g}$ of 4-dimethylaminopyridine (DMAP) (1/300 of the amount of LacOCA) was added and stirred at room temperature overnight until no bubbles were present. Once the reaction was complete, the solution was concentrated and dried at $30^{\circ} \mathrm{C}$. Then, chloroform was used to dissolve the polymerization products. At room temperature for $12 \mathrm{~h}$, the insoluble substances were removed by centrifugation. The filtrate of homogeneous phase was precipitated by excessive ethanol to achieve solidity. The solids were filtered and dried under vacuum at $60^{\circ} \mathrm{C}$ for $8 \mathrm{~h}$. The product gained was GO-g-PLLA (Scheme 1). As a contrast, PLA and GO are also dissolved in chloroform, and at room temperature for $12 \mathrm{~h}$, the insoluble substances were removed by centrifugation. The filtrate of homogeneous phase was precipitated by excessive ethanol to achieve solidity. The solids were filtered and dried under vacuum at $60{ }^{\circ} \mathrm{C}$ for $8 \mathrm{~h}$. The product gained was not GO/PLLA 
blend compound, it was PLA. Filtration after centrifugation was dried using freeze-drying method to obtain GO.

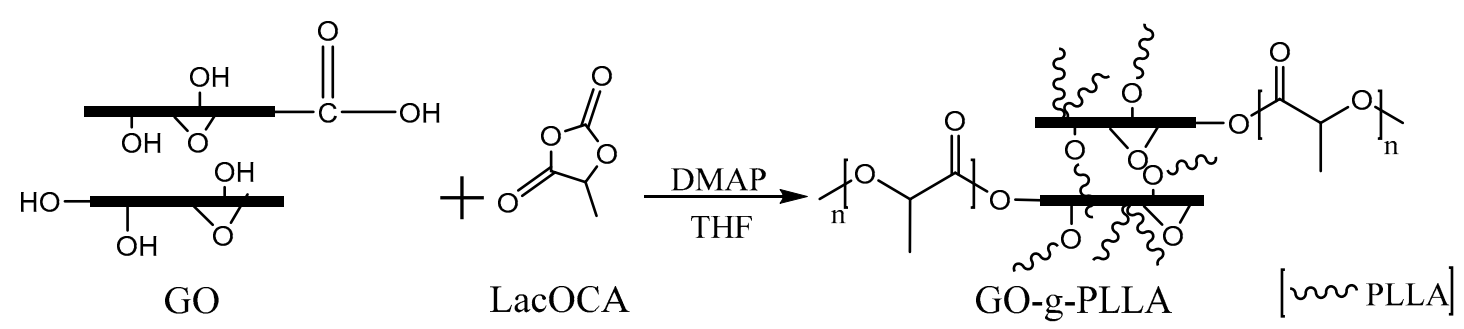

Scheme 1. Synthetic route of graphene oxide (GO) grafted with poly L-lactic acid molecular chains (GO-g-PLLA).

\subsubsection{Preparation of GO-g-PLLA/PLLA Composite Material}

A specific amount of GO-g-PLLA $(4,8$, or $12 \mathrm{~g})$ was mixed with $800 \mathrm{~g}$ of PLLA. Chloroform $(2000 \mathrm{~mL})$ was then added and mechanically stirred to dissolve the mixture. After the solution became clear, the solution was sonicated for $2 \mathrm{~h}$. Then, $5000 \mathrm{~mL}$ of ethanol was added to the solution to precipitate a gray-white solid that was vacuum-dried at $60{ }^{\circ} \mathrm{C}$ for $8 \mathrm{~h}$ to obtain GO-g-PLLA/PLLA composites containing $0.5 \%, 1 \%$, and $1.5 \%$ GO-g-PLLA.

\subsubsection{Instruments and Characterization}

Nuclear magnetic resonance (NMR), infrared (IR), TGA, and X-ray photoelectron spectroscopy (XPS) were conducted to determine the changes in the chemical characteristics of GO and GO-g-PLLA. NMR spectra were recorded with Bruker $400 \mathrm{MHz}$ spectrometers at $25^{\circ} \mathrm{C}$. Chemical shifts for ${ }^{1} \mathrm{H}$ NMR spectra were referenced internally using the residual solvent resonances and tetramethylsilane (TMS) as an internal reference. The solvent was used in the deuterium chloroform, and with a solubility of $10 \mathrm{mg} / \mathrm{mL}$. the dissolution was not precipitation. it was limpid. The molecular weight of free PLLA was determined using gel permeation chromatography (GPC) at $40^{\circ} \mathrm{C}$ using THF as the eluent with a flow rate of $1 \mathrm{~mL} / \mathrm{min}$. The system equipped with a Waters 515 pump and a Waters 2414 refractive index detector. IR (Bruker Tensor 37) spectra were recorded from 600 to $4000 \mathrm{~cm}^{-1}$ with a resolution of $2 \mathrm{~cm}^{-1}$ and 32 scans. TGA curves were obtained from a Mettler SDTA851e. The samples were heated from 25 to $800{ }^{\circ} \mathrm{C}$ at a rate of $10^{\circ} \mathrm{C} / \mathrm{min}$ in an aluminum crucible under $50 \mathrm{~mL} / \mathrm{min}$ of nitrogen purging. XPS measurements were performed using a PHI5000 Versaprobe-II System Auger electron spectrometer (Physical Electronics Company, U.S.) equipped with a hemispherical electron analyzer and a scanning monochromatic AlKa $(\mathrm{hm}=1486.6 \mathrm{eV}) \mathrm{X}$-ray source. The particle size distributions of GO and GO-g-PLLA in the chloroform were tested using a S3500SI laser particle size analyzer system (Micortrac Co. Ltd., Norristown, PA, USA) with an equivalent sphere model and a measuring range of 0.01 to $2800 \mu \mathrm{m}$. To further characterize the dispersions of GO and GO-g-PLLA before and after grafting in solvents, we performed dynamic light scattering to measure the particle size and distribution. The solutions had a concentration of $1 \mathrm{mg} / \mathrm{mL}$. Each solution was sonicated for $2 \mathrm{~h}$ after dissolving the particles and immediately tested.

The crystallization behaviors of PLLA and nanocomposites were measured by a differential scanning calorimeter (DSC) (214, Netzsch, Selb, Germany) in a nitrogen flow (50 $\mathrm{mL} / \mathrm{min}$ ) with a heating rate of $10{ }^{\circ} \mathrm{C} / \mathrm{min}$ over a temperature range of 20 to $200{ }^{\circ} \mathrm{C}$. Flexural tests of the PLLA composites were determined by a CMT4104 universal tester (Meites Industrial Systems Co., Ltd. (Wuhu, China)). The length, width, and thickness of the sample were 50, 15 and $1.10 \mathrm{~mm}$, respectively, according to GB/T 1040.3-2006 standard, at a crosshead speed of $50 \mathrm{~mm} / \mathrm{min}$. Five different measurements were recorded for each sample. The Water Vapor Permeability (WVP) of the films was gravimetrically determined according to the ASTM E96-95 standard. The films had a thickness of 
$0.04 \pm 0.005 \mathrm{~mm}$ and were obtained by casting. The screw insertion temperatures were $160,175,175$, and $170{ }^{\circ} \mathrm{C}$. The $\mathrm{PO}_{2}$ was tested on an Oxy Sense5250I oxygen analyzer.

\section{Results and Discussion}

\subsection{Characterization of GO-g-PLLA}

Chaobin and Inoue reported the synthesis of GO-g-PLLA through the ring-opening polymerization of lactide monomers, initiated by the grafted $\mathrm{OH}$ groups on $\mathrm{GO}$ and under the catalysis of $\mathrm{Sn}(\mathrm{Oct})^{2}$. The synthesis temperature was $120^{\circ} \mathrm{C}$ and a metal catalyst was used $[10,15]$. Considering the biomedical and food potential of PLLAs, studies have focused on metal-free synthetic methods under mild conditions. Bourissou et al. reported the metal-free synthesis of PLLA by ring-opening polymerization of LacOCA. LacOCA exhibited remarkable reactivity compared to lactide. PLLA of controlled molecular weights and narrow polydispersities are typically obtained under mild conditions using DMAP and various protic initiators [16-19]. The above studies demonstrated the ring-open polymerization of lactide or LacOCA. When there are hydroxyl compounds in the polymerization system, after polymerization, these hydroxyl compounds are linked to PLLA chains through chemical bonds to achieve the modification of PLLA [10,17-19]. In this study, we use metal-free synthetic methods to synthesize GO-g-PLLA by ring-opening polymerization of LacOCA, as shown in Figure 1.

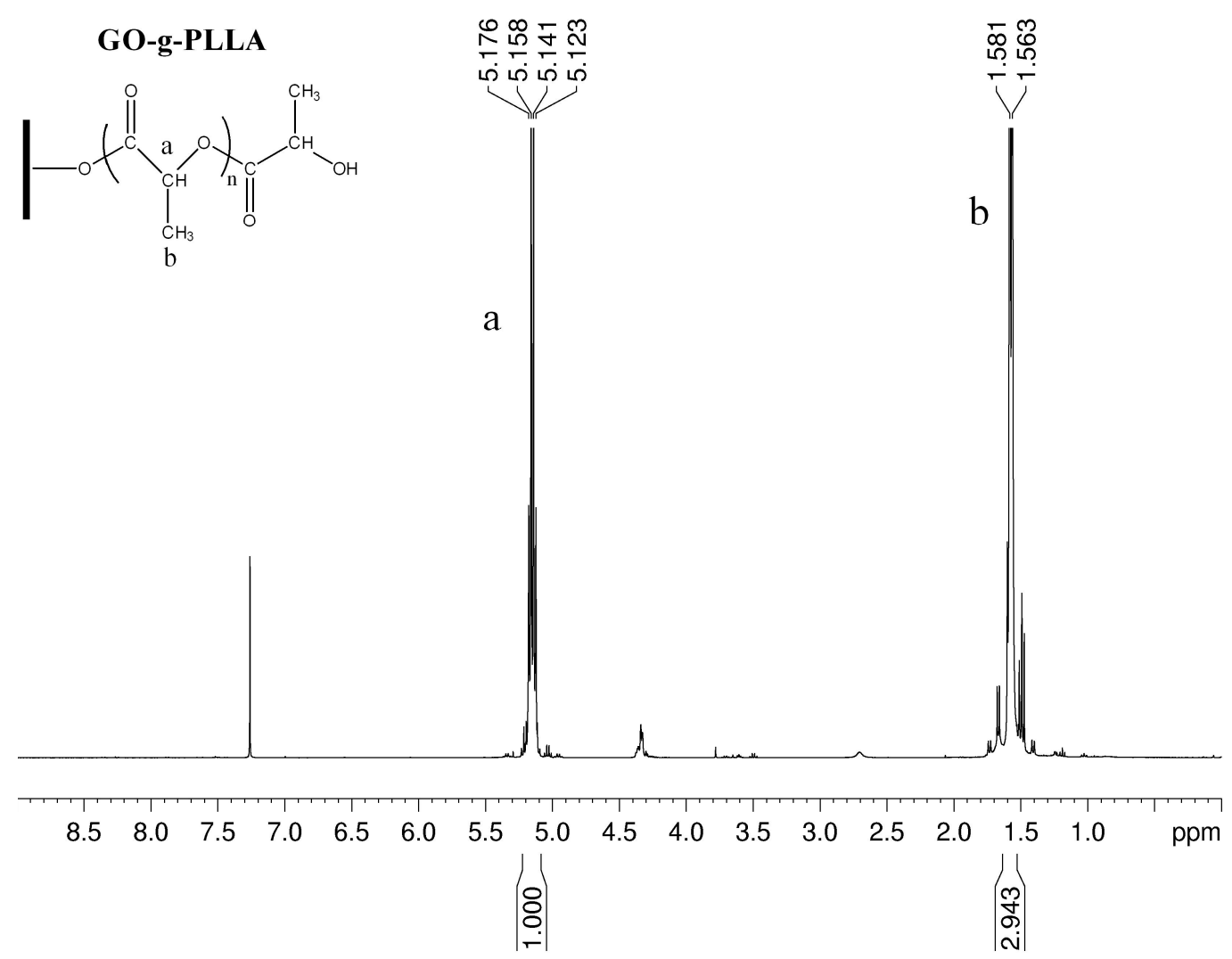

Figure 1. The $400 \mathrm{MHz}{ }^{1} \mathrm{H}$ nuclear magnetic resonance (NMR) spectrum of the GO-g-PLLA.

In our experiment with the preparation of GO-g-PLLA, the polymerization was completed. Then chloroform was used to dissolve the polymerization products. After $12 \mathrm{~h}$ of placement, we found that the chloroform solution was homogeneous. No insoluble substance was found by centrifugation. The filtrate of homogeneous phase was precipitated by excessive ethanol to achieve solidity. The solids were filtered and dried under vacuum to obtain GO-g-PLLA. In the comparison experiment of 
preparing GO/PLLA blend compound, we found that the chloroform dissolved in GO/PLLA after $12 \mathrm{~h}$ statics was divided into two phases. Because GO is insoluble in chloroform, it will be precipitated from the solution. After centrifuge separation, the insoluble substance obtained is pure GO. The compounds dissolved in chloroform were found to be pure PLA after the same process. The two experiments also showed that free GO could be separated from GO- $g$-PLLA or PLLA by chloroform dissolution and centrifugation. If GO/PLLA blend compound is to be prepared, the GO and PLA cannot be centrifuged after mixing in chloroform; GO/PLLA can be obtained by direct freeze-drying.

To confirm that the GO-g-PLLA has a PLA structure unit, we performed magnetic characterization on the GO-g-PLLA polymers. From Figure 1, the characteristic peaks of PLLA appeared at 1.5 and $5.1 \mathrm{ppm}$, which agree with data reported in the literature [20-25] and belong to the characteristic proton peaks for the methine (a) and methyl (b) groups on the PLLA chain. From the NMR spectrum of the GO-g-PLLA, 4.35 ppm corresponds to the methine group - $\mathrm{CH}$ - located at the end of the PLLA chain, which is adjacent to the terminal hydroxyl groups of PLLA. This finding is consistent with that of Sun and He [10], in which GO-g-PLLA was prepared by the ring-opening polymerization of lactide.

To confirm that the GO-g-PLLA has a GO structure unit, we performed XRD on the GO-g-PLLA polymers. Figure 2 shows the XRD patterns of pure GO, PLLA, and GO- $g$-PLLA composite. As seen, the most intense diffraction peak of PLLA at $2 \theta=16.6^{\circ}$, the diffraction peak of GO at $2 \theta=10.5^{\circ}$. For the GO-g-PLLA composite, the location and shape of the diffraction peaks of the three samples were similar to those of pure PLLA and GO. As the sample was dissolved by chloroform and separated by centrifuge, the results thus show that the PLLA molecular chains were successfully grafted to the surface of GO.

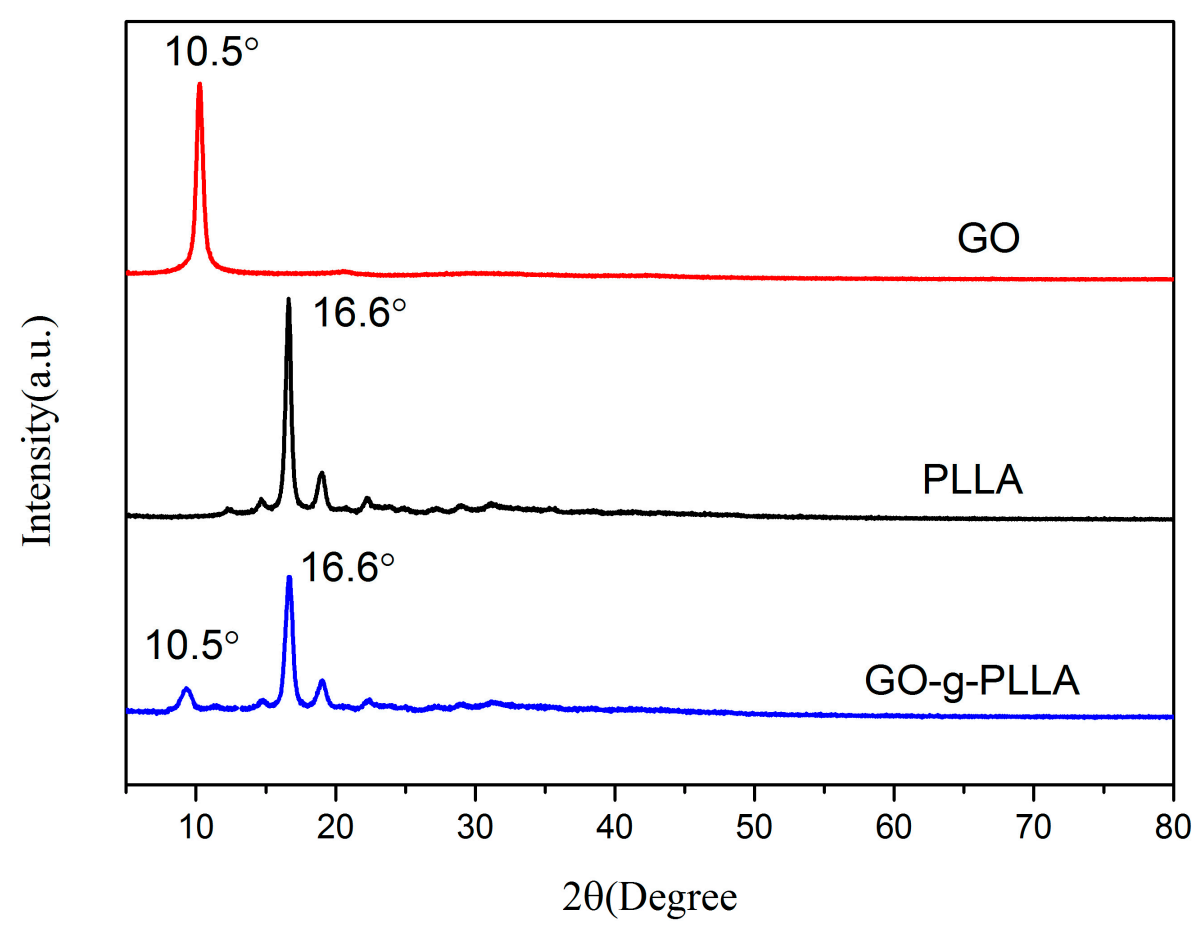

Figure 2. The XRD patterns of pure GO, PLLA, and GO- $g$-PLLA.

In order to further confirm that the GO-g-PLLA has a GO structure unit, we performed UV/Vis characterization on the GO-g-PLLA polymers. Figure 3 shows the UV/Vis spectra of PLLA, GO, GO- $g$-PLLA, and GO/PLLA. The UV/Vis spectrum of PLLA, GO- $g$-PLLA, and GO/PLLA was observed in the DCM solution, while those of PLLA, GO-g-PLLA, and GO/PLLA were in the solution. GO-g-PLLA shows very broad absorption with continuously decreasing intensity ranged from 220 to $330 \mathrm{~nm}$. On the other hand, PLLA and GO-g-PLLA shows the absorption in the range 
from 250 to $330 \mathrm{~nm}$, and no absorption peak is observed in the 330 to $800 \mathrm{~nm}$ range. Further, PLLA, GO-g-PLLA, and GO/PLLA shows characteristic peaks in the wave length region shorter than $250 \mathrm{~nm}$, while no evident absorption in the higher wave length region. In the absorption spectrum in the range from 220 to $320 \mathrm{~nm}$, the GO-g-PLLA shows absorption with special features characteristics for both PLLA and GO, indirectly indicating that the PLLA chain was grafted onto the surface of GO [15].

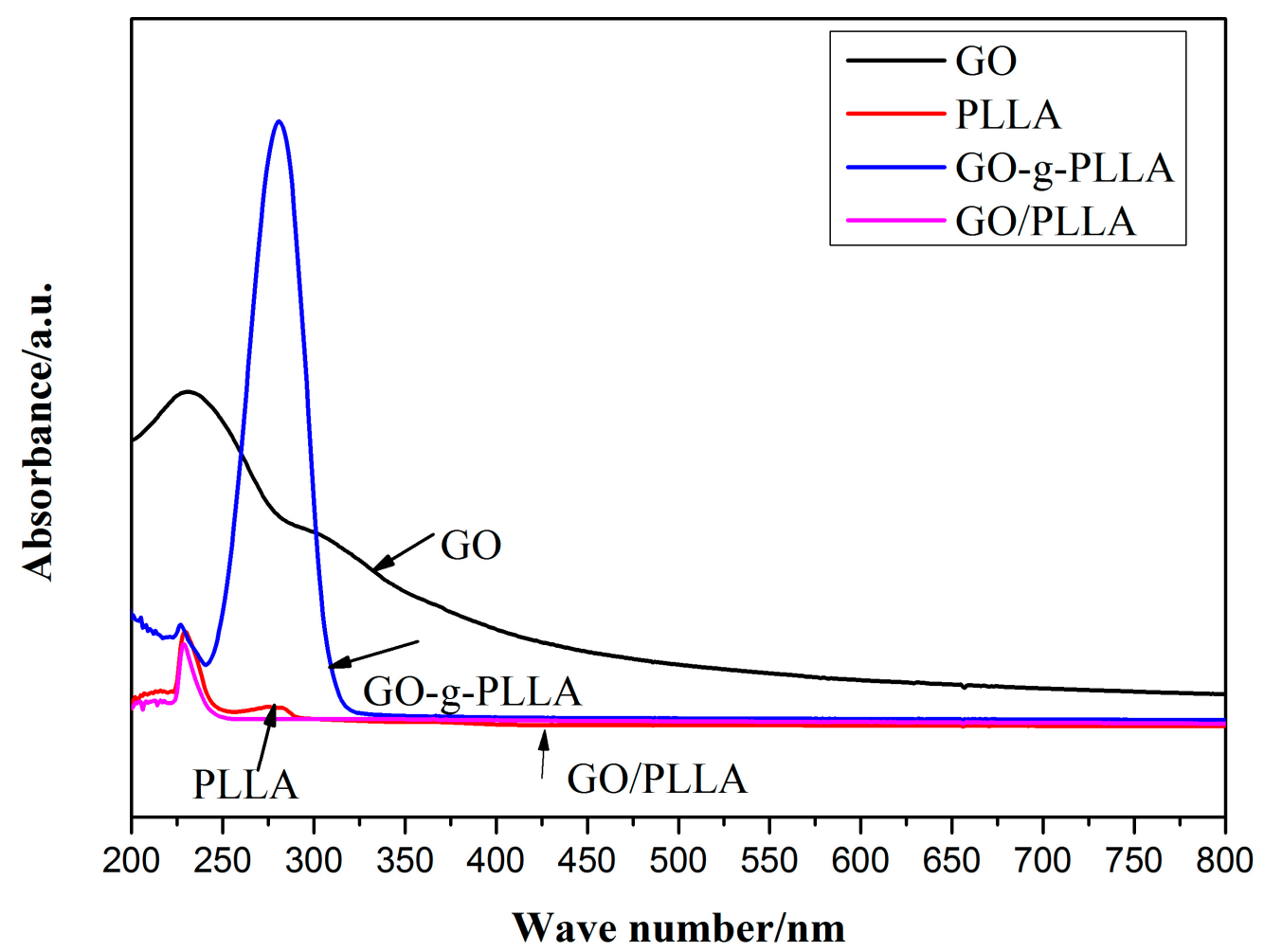

Figure 3. UV/Vis spectra of GO, PLLA, GO- $g$-PLLA, and GO/PLLA.

\subsection{Determination of Molecular Weight by Gel Permeation Chromatography (GPC)}

We estimated the grafting by testing the molecular weight of the free PLLA generated in the reaction of the molecular length of PLLA to the graphene surface $[26,27]$. The GO-g-PLLA polymer had sharp, unimodal distributions, indicating that GO had completely copolymerized with lactide and that no lactide homopolymerization had occurred. The average molecular weight was 15,000 g/mol, and the polydispersity coefficient was 1.09 .

\subsection{Infrared (IR) Spectroscopy}

Figure 4 shows the Fourier transform IR (FTIR) spectra of the polymers, in which the typical polyester absorption peaks appeared. In addition, the PLLA, GO, and GO-g-PLLA IR measurements detected changes in the chemical functional groups upon grafting, as shown in Figure 4 . The peaks at 3447,3440 , and $3443 \mathrm{~cm}^{-1}$ belong to the O-H stretching peaks in PLLA, GO, and GO-g-PLLA, respectively. The peak at $1758 \mathrm{~cm}^{-1}$ corresponds to the stretching vibration peak of $\mathrm{C}=\mathrm{O}$ in the ester bond in the PLLA chain (Figure $4 a, d)[28,29]$. A significantly stronger $C=O$ stretching vibration peak appeared at $1758 \mathrm{~cm}^{-1}$ for GO-g-PLLA, which was likely caused by the grafting of the PLLA molecular chains onto the surface of GO. Therefore, the hydroxyl groups on the surface of GO initiated the ring-opening of the lactic acid $O$-carboxyanhydride and the esterification with carboxyl groups [30]. The GO/PLLA blends were also tested by infrared contrast, and the GO/PLLA curves of the blends were found to be different from those of the copolymer (Figure $4 \mathrm{~d}$ ). Figure $4 \mathrm{~d}$ is consistent with the findings of Sun and He [10], in which GO-g-PLLA was prepared by the ring-opening polymerization 
of lactide. Furthermore, the characteristic peaks of PLLA, including the stretching vibration of $\mathrm{C}-\mathrm{CH}_{3}$, the bending vibration of $-\mathrm{CH}_{3}$, and the asymmetric bending vibration of $-\mathrm{CH}_{3}$, appeared at 1093,1185 , and $1457 \mathrm{~cm}^{-1}$, respectively $[15,28,31-33]$, in the spectrum of GO-g-PLLA. The results thus show that the PLLA molecular chains were successfully grafted onto the surface of GO.

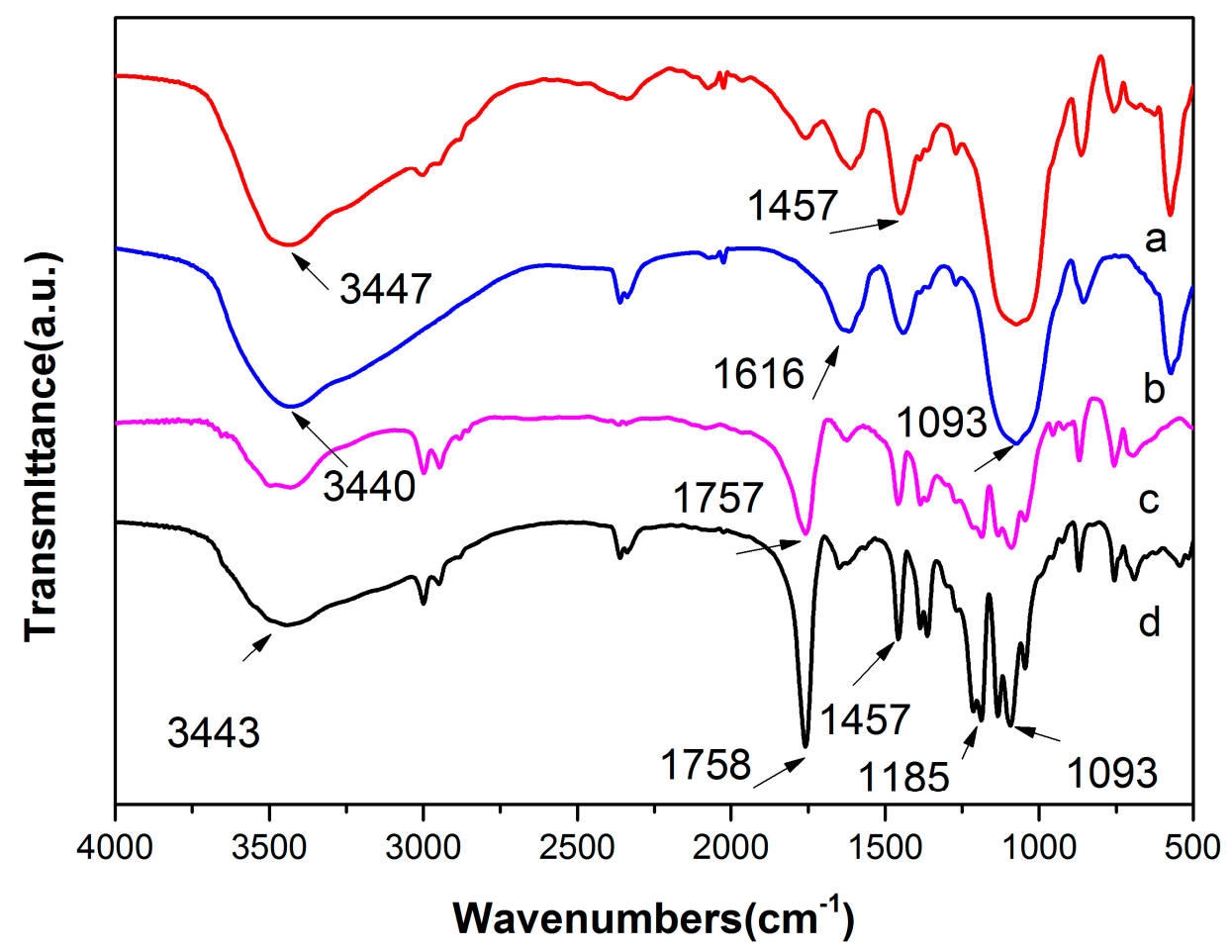

Figure 4. Fourier transform IR (FTIR) spectra of (a) PLLA, (b) GO, (c) GO/PLLA, and (d) GO-g-PLLA.

\subsection{Thermogravimetric Analysis (TGA)}

From Figure 5, the weight loss of GO appeared in the temperature range of 50 to $200{ }^{\circ} \mathrm{C}$. The weight loss at $50-100{ }^{\circ} \mathrm{C}$ was due to the evaporation of physical water in GO, and the weight loss at $100-200{ }^{\circ} \mathrm{C}$ was caused by the decomposition of hydroxyl and carboxyl groups on the surface of GO to carbon monoxide (CO), carbon dioxide $\left(\mathrm{CO}_{2}\right)$, and water vapor [34-36]. The weight loss of GO-g-PLLA appeared at $220-380^{\circ} \mathrm{C}$, which can be divided into two regions. The first region that occurred at $220-280{ }^{\circ} \mathrm{C}$ was attributed to the degradation of the residual oxygen-containing functional groups on GO; the other region at $280-380^{\circ} \mathrm{C}$ was caused by the degradation of PLLA grafted to the surface of GO $[26,34,35]$. The PLLA weight loss mainly appeared at $340-380^{\circ} \mathrm{C}$. Based on the literature and according to the TGA curves, the grafting ratio of PLLA to the surface of GO was approximately $60.2 \mathrm{wt} \%$ [37] for the in-situ ring-opening polymerization of the lactic acid $O$-carboxyanhydride. The weight loss of GO-g-PLLA was between those of GO and PLLA. We also performed a TG analysis on GO/PLLA blends, and the results showed that a significant difference existed compared to the copolymer of GO-g-PLLA. The results thus show that the PLLA molecular chains were successfully grafted to the surface of GO.

\subsection{X-ray Photoelectron Spectroscopy (XPS)}

To further characterize the changes in the chemical functional groups on GO, we performed XPS before and after grafting. The $\mathrm{C} 1 \mathrm{~s}$ peaks before and after grafting are shown in Figure 6 . The areas of the peaks for GO before grafting were as follows: the $\mathrm{C}=\mathrm{C} / \mathrm{C}-\mathrm{C}$ peak $(283.38 \mathrm{eV})$ was $26.1143 \%$, the $\mathrm{C}-\mathrm{OH}$ peak $(284.21 \mathrm{eV})$ was $16.7404 \%$, the $\mathrm{C}-\mathrm{O}$ peak $(285.75 \mathrm{eV})$ was $37.8132 \%$, the $\mathrm{C}=\mathrm{O}$ peak $(286.47 \mathrm{eV})$ was $13.7880 \%$, and the $\mathrm{O}-\mathrm{C}=\mathrm{O}$ peak $(287.41 \mathrm{eV})$ was $5.5441 \%[38,39]$. The $\mathrm{C} 1$ s peaks 
after grafting are shown in Figure $4 \mathrm{~b}$. The peak area of -OH for GO changed to $5.5441 \%$, exhibiting a substantial decrease. The peak areas of $\mathrm{C}-\mathrm{H}$ and $\mathrm{O}-\mathrm{C}=\mathrm{O}$ substantially increased to $17.2537 \%$ and $16.8664 \%$, respectively [36]. The results show that, as the initiator, the hydroxyl groups $(-\mathrm{OH})$ on the surface of GO initiated the ring-opening polymerization of LacOCA with the assistance of a catalyst, thus grafting the polymer to the surface of GO [40]. As the reaction progressed, the surface of GO was almost completely covered by a layer of PLLA molecular chains. The test results show that the carbon to oxygen ratio decreased from 2.16 for GO to 1.67 for GO-g-PLLA. This is because PLLA contains more oxygen that GO, which agrees with the reports in the literature [37].

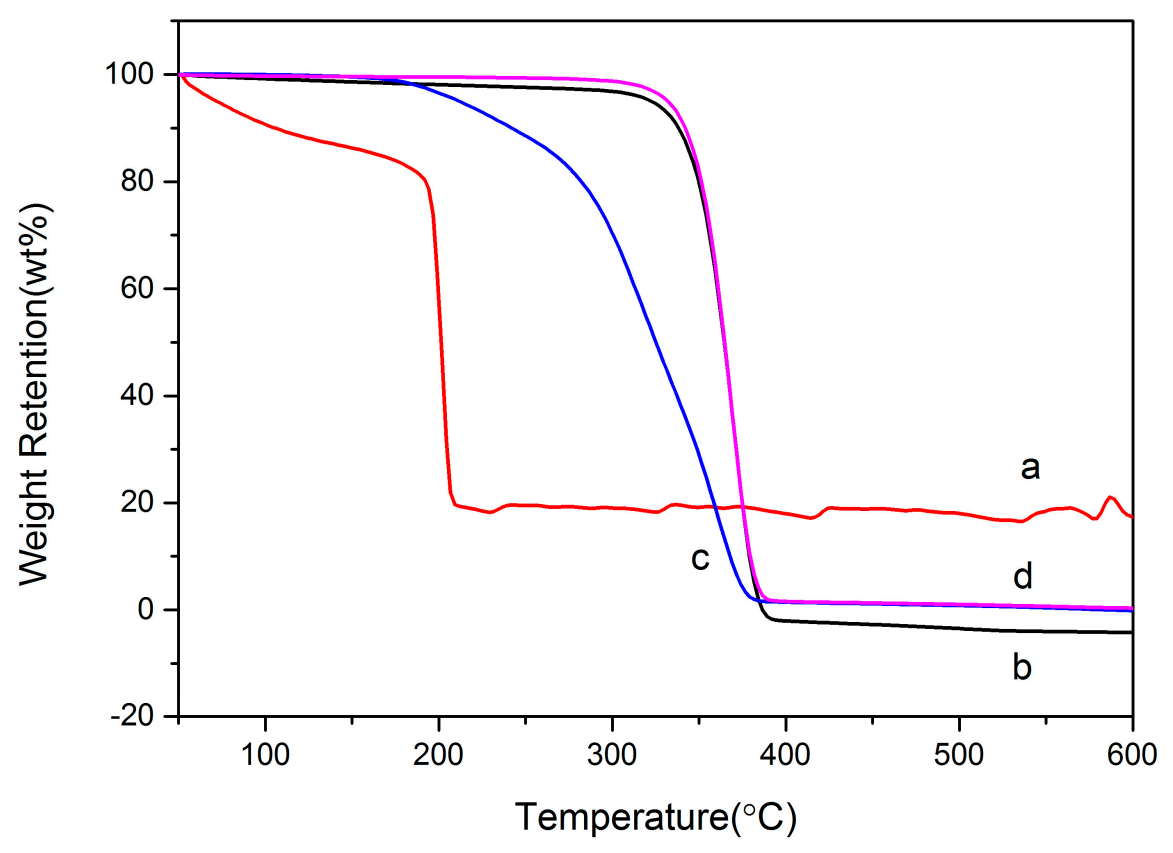

Figure 5. TGA curves of (a) GO, (b) PLLA, (c) GO-g-PLLA, and (d) GO/PLLA.
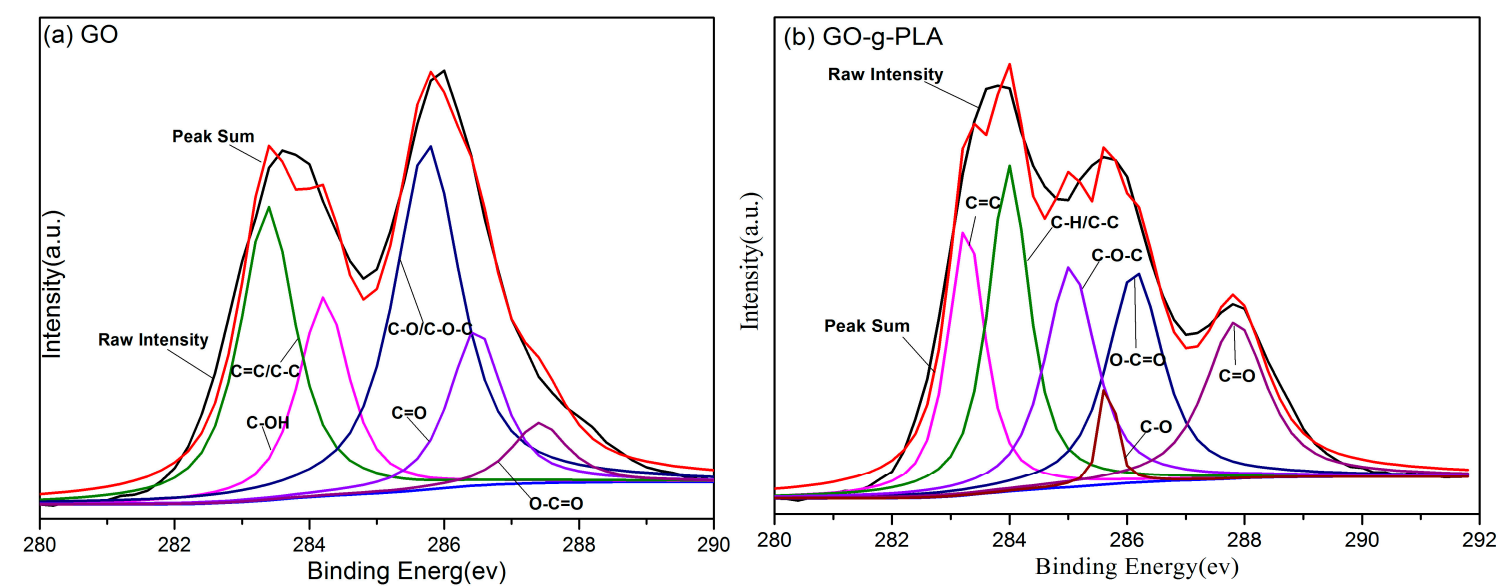

Figure 6. X-ray photoelectron spectroscopy (XPS) curves of (a) GO and (b) GO-g-PLLA. 


\subsection{Characterization of Physical Properties}

To further characterize the dispersions of GO and GO-g-PLLA before and after grafting in solvents, we used dynamic light scattering to measure the particle size and distribution. The solutions had a concentration of $1 \mathrm{mg} / \mathrm{mL}$. Each solution was sonicated for $2 \mathrm{~h}$ after dissolving the particles and immediately tested. The results (Figure 7) show that the particle diameters of GO and were $108.5 \mu \mathrm{m}$ in $\mathrm{H}_{2} \mathrm{O}$ and $163.8 \mu \mathrm{m}$ in chloroform. Thus, the dispersion of GO in chloroform was significantly better than in water. The results indicate that GO existed as a stable single layer or as few layers in water [41]. Because of the strong van der Waals' force between the GO nanosheets, GO tends to agglomerate. Therefore, the particle size and size distribution of GO in solvents can reflect its dispersion in solvents [42]. The particle size of GO-g-PLLA dissolved in water was $81.8 \mu \mathrm{m}$, exhibiting a very wide particle size dispersion. The results show that after the ring-opening polymerization of lactic acid $O$-carboxyanhydride, the hydrophobic PLLA was successfully grafted onto the surface of GO, thus making GO-g-PLLA a hydrophobic material, exPLLAining its poor dispersion in water. A particle size of $30.59 \mu \mathrm{m}$ in chloroform indicated that the dispersion of GO- $g$-PLLA in chloroform was substantially better than that in water. After grafting, GO was transformed from a hydrophilic into a hydrophobic material. Therefore, improving the dispersion of GO in chloroform is possible by obtaining functional GO- $g$-PLLA through the ring-opening polymerization of lactic acid $\mathrm{O}$-carboxyanhydride initiated by the hydroxyl groups on the GO surface.

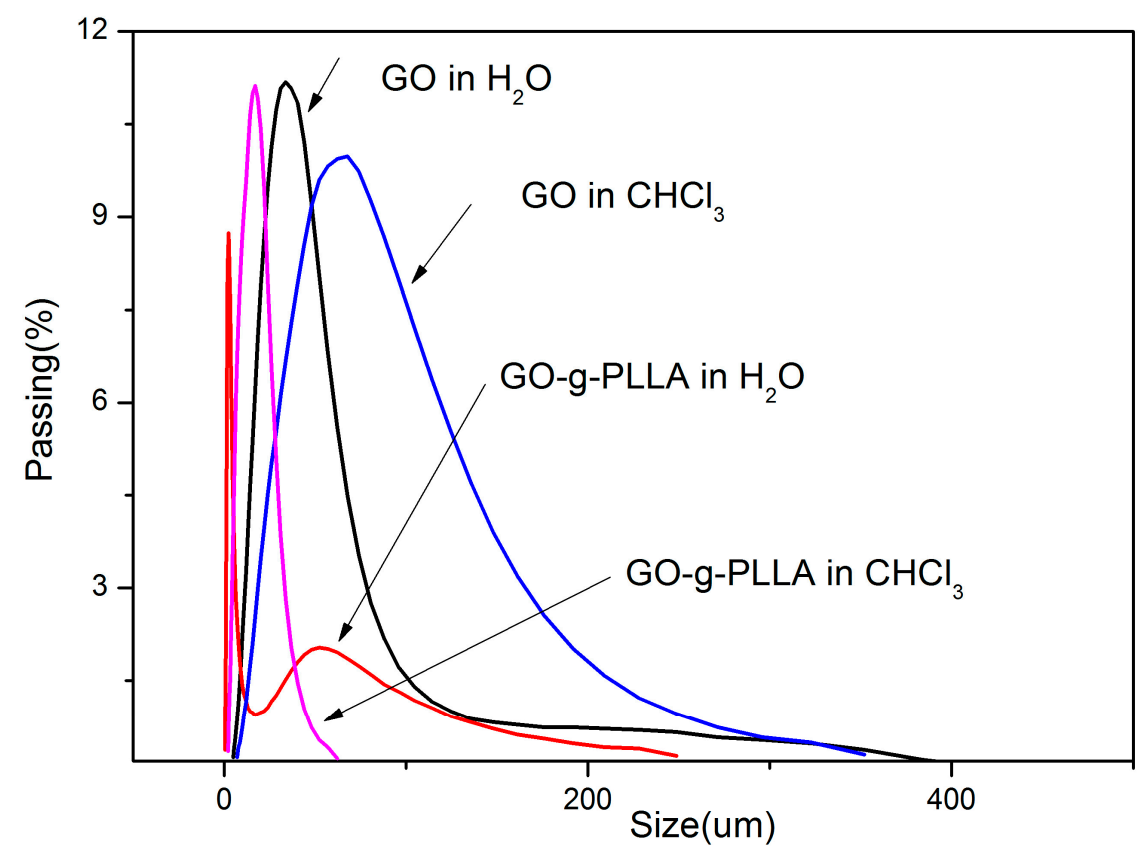

Figure 7. Laser size and shape analysis of GO and GO- $g$-PLLA.

To further confirm the solubility of GO and GO- $g$-PLLA in chloroform, we prepared $0.5 \mathrm{mg} / \mathrm{mL}$ $\mathrm{GO}$ and GO-g-PLLA solutions in chloroform. After the solutions were sonicated for $2 \mathrm{~h}$ and left standing for $12 \mathrm{~h}$ (Figure 8), the solubility was investigated. All the GO precipitated to the bottom of the chloroform after the solution was sonicated and left for $12 \mathrm{~h}$, whereas GO-g-PLLA was still evenly dispersed in the chloroform. Similarly, when chloroform was used to dissolve GO and PLLA blends, $12 \mathrm{~h}$ later, GO was precipitated, because the dispersion of GO in chloroform is poor. These different behaviors indicate that the PLLA molecular chains grafted to the GO surface very strongly interacted with the solvent, thus increasing the dispersion of GO-g-PLLA in chloroform [37]. 

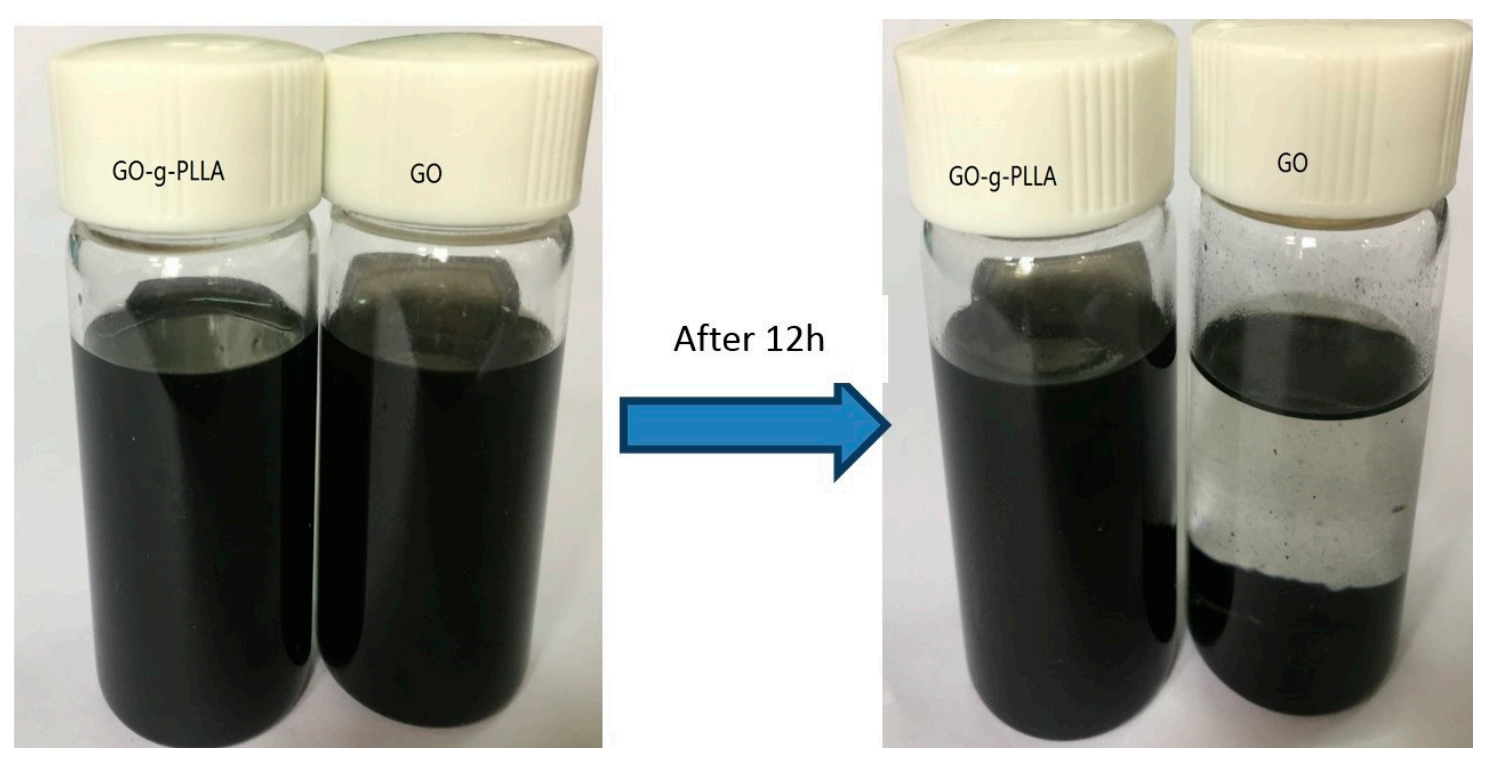

Figure 8. Dispersions of GO and GO-g-PLLA in chloroform.

\subsection{Differential Scanning Calorimetry (DSC) of PLLA and Its Composites}

From the DSC curves in Figure 9 and Table 1, the glass transition $(\mathrm{Tg})$ and melting $(\mathrm{Tm})$ temperatures of pure PLLA are 63 and $167.8^{\circ} \mathrm{C}$, respectively. Tg and Tm increased to 68 and $168{ }^{\circ} \mathrm{C}$, respectively, after adding $0.5 \%$ GO-g-PLLA. Tm increased to $170{ }^{\circ} \mathrm{C}$ after adding $1 \%$ and $1.5 \%$ GO-g-PLLA. However, Tg did not increase markedly with further increases in GO-g-PLLA content. When $1.5 \%$ GO-g-PLLA was added, Tg decreased to $62{ }^{\circ} \mathrm{C}$. The increases in $\mathrm{Tg}$ and $\mathrm{Tm}$ for the nanocomposite were likely due to the increase in the interaction between PLLA and GO and its derivatives. The mechanical interlocking, hydrogen bonding, and/or electrostatic forces restrict the movement between the polymer chains $[43,44]$. Therefore, the composite with GO-g-PLLA exhibited better thermal stability and a higher interfacial adhesion strength than pure PLLA.

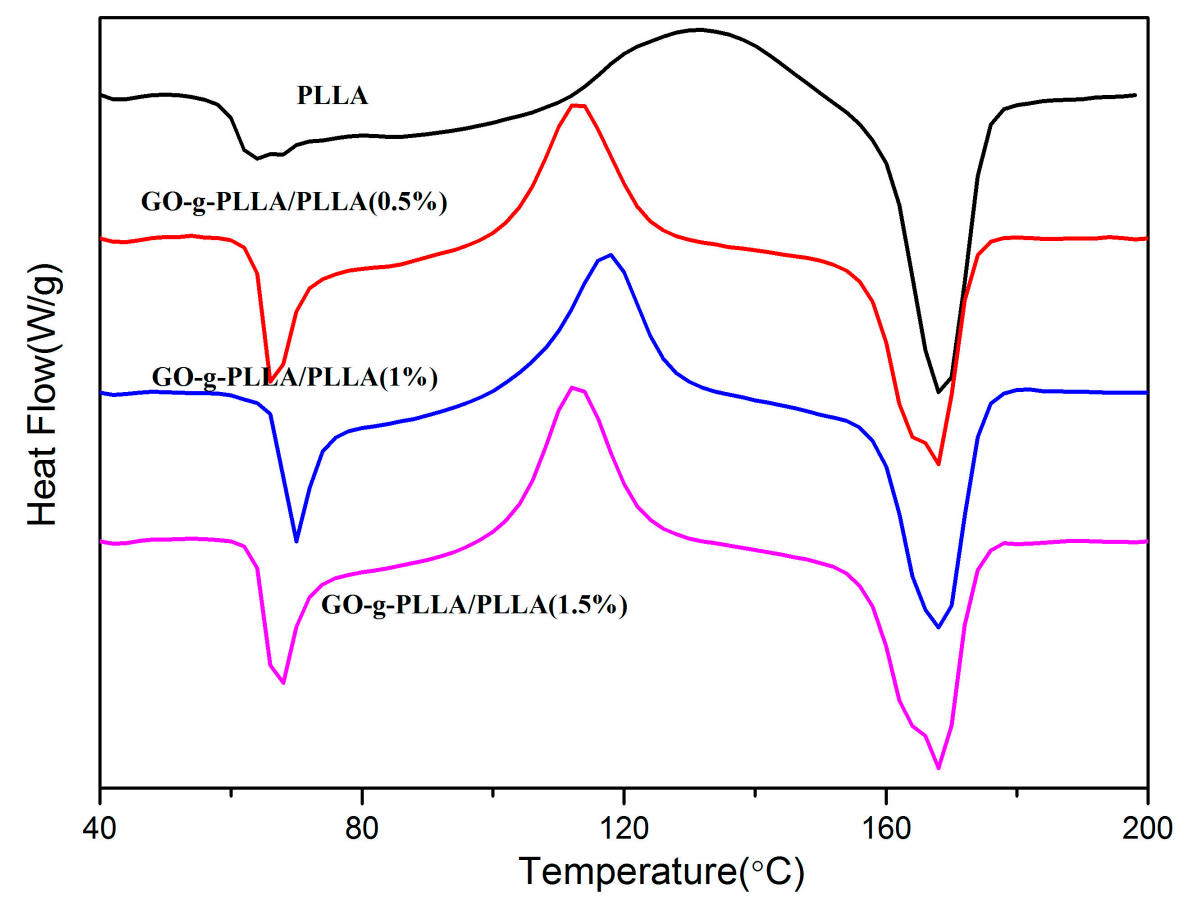

Figure 9. Differential scanning calorimetry (DSC) curves of PLLA and GO-g-PLLA/PLLA. 
Table 1. The glass transition temperature $(\mathrm{Tg})$, melting temperature $(\mathrm{Tm})$, enthalpy of melting $(\Delta \mathrm{Hm})$, and degree of crystallinity $\left(\mathrm{Xc}_{\mathrm{c}}\right)$ of PLLA and GO-g-PLLA/PLLA.

\begin{tabular}{ccccc}
\hline Sample & $\mathbf{T g}\left({ }^{\circ} \mathbf{C}\right)$ & $\operatorname{Tm}\left({ }^{\circ} \mathbf{C}\right)$ & $\Delta \mathbf{H m}(\mathbf{J} / \mathbf{g})$ & $\mathbf{X c}(\mathbf{\%})$ \\
\hline PLLA & 63 & 167.8 & 23.84 & 25.6 \\
GO-g-PLLA/PLLA $(0.5 \%)$ & 68 & 168 & 35.67 & 38.3 \\
GO-g-PLLA/PLLA $(1 \%)$ & 68 & 170 & 39.51 & 42.4 \\
GO-g-PLLA/PLLA $(1.5 \%)$ & 62 & 170 & 37.13 & 39.8 \\
\hline
\end{tabular}

In addition, DSC data showed that the addition of the GO-g-PLLA composite improved the crystallization rate of PLLA, as shown in Table 1 , in which $\Delta \mathrm{Hm}(\mathrm{J} / \mathrm{g})$ and $\mathrm{Xc}_{\mathrm{c}}$ represent the enthalpy of melting and degree of crystallinity, respectively. Thus, with the addition of $0.5 \%$ GO-g-PLLA, Xc increased from $25.6 \%$ to $38.3 \%$ and continued to increase with increasing amounts of GO-g-PLLA composite.

\subsection{Characterization of the Mechanical Properties of PLLA and Its Composite}

Figure 10 shows the tensile strength and breaking strain of PLLA, GO/PLLA, and GO-g-PLLA/PLLA. The tensile test results show that the tensile strength and breaking strain of PLLA were $47.97 \mathrm{MPa}$ and $4.44 \%$, respectively, which increased to $51.09 \mathrm{MPa}$ and $5.64 \%$ after adding GO and further increased to $71.26 \mathrm{MPa}$ and $6.17 \%$ after adding the GO-g-PLLA composite. Both copolymers and blends enhance the mechanical properties of PLLA. The GO-g-PLLA composite exhibited increased tensile strength and breaking strain of $48.6 \%$ and $39 \%$, respectively. The results confirm those of previous reports because of the high specific surface area and high elastic modulus. After dispersion in polymers, graphene and its derivatives can bear loads and thus considerably enhance the mechanical properties of a polymer [45-47].

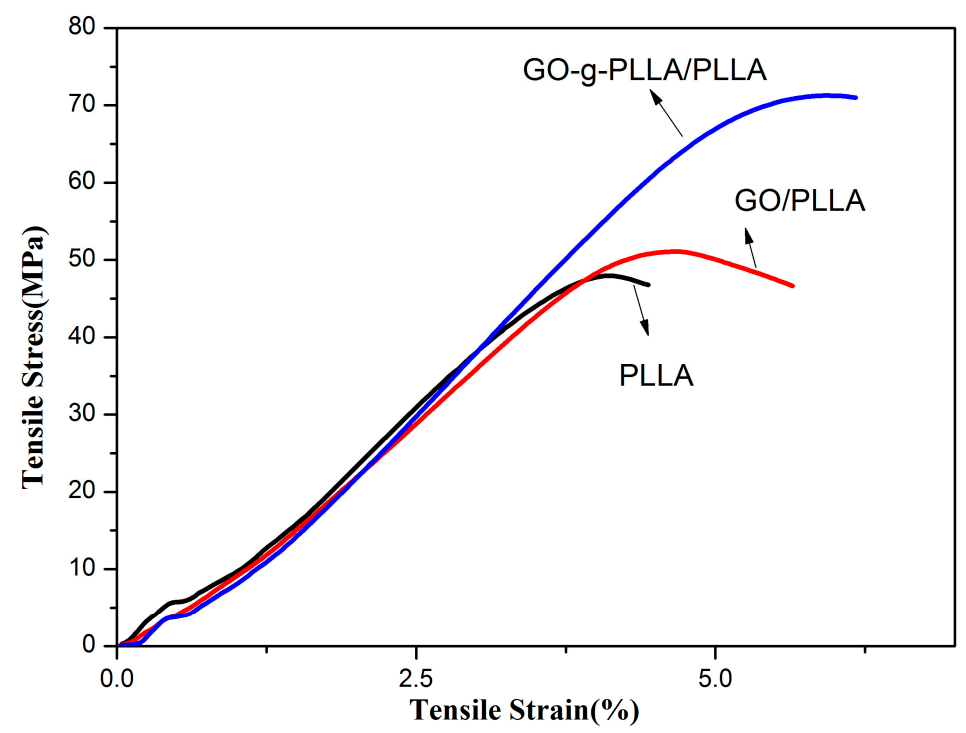

Figure 10. Stress-strain curves of PLLA, GO/PLLA, and GO- $g$-PLLA/PLLA.

\subsection{Characterization of the Morphology of PLLA and Its Composite}

To investigate the dispersion of GO in the composite and the morphology of the fracture surface, we used scanning electron microscopy (SEM) to characterize the fracture surface of PLLA and GO-g-PLLA/PLLA, as shown in Figure 11. A large quantity of evident undulations appeared at the fracture surface of PLLA (Figure 11a). In contrast, for GO-g-PLLA/PLLA (Figure 11b), the fracture surface was coarser and denser than that of PLLA, indicating that the energy required for fracturing 
is higher than that for pure PLLA. In addition, the fracture of the composite was different from the brittle fracture of pure PLLA. Conversely, there was no clear agglomeration of graphene on the fracture surface, further indicating that GO-g-PLLA was evenly dispersed in PLLA.

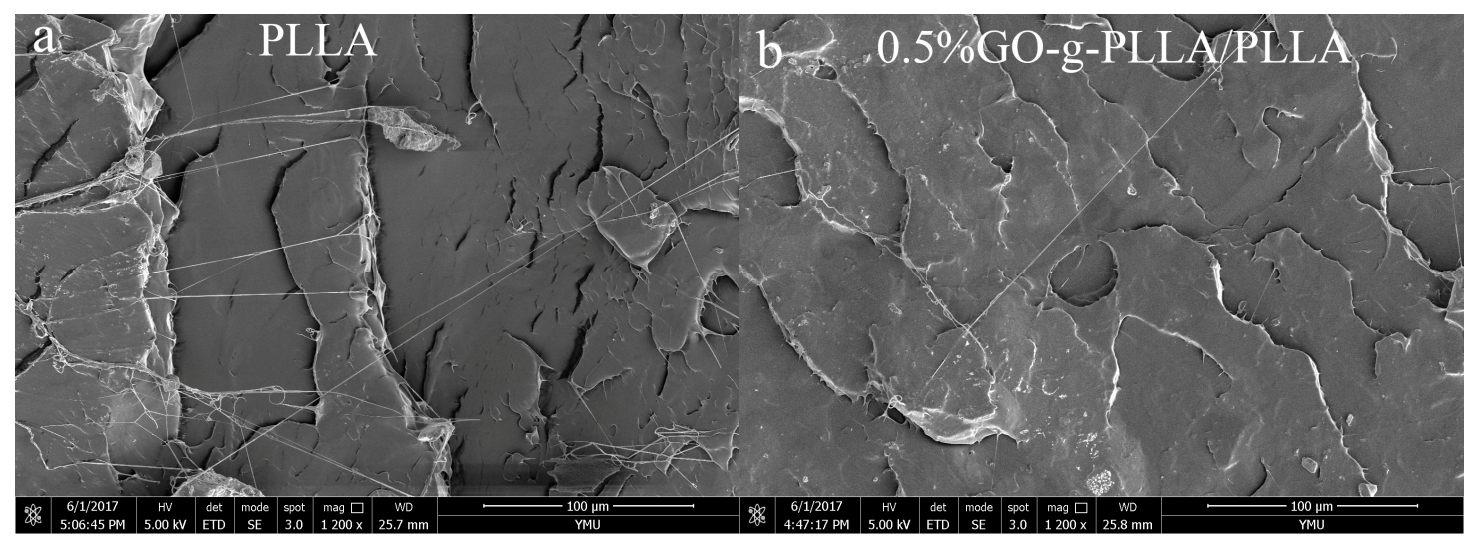

Figure 11. Scanning electron microscopy (SEM) of the tensile fracture surfaces of PLLA and GO-g-PLLA/PLLA.

\subsection{Barrier Tests}

\subsubsection{Water Vapor Permeability (WVP) Test}

WVP is the most important barrier property for materials. The WVP values of pure PLLA and its nanocomposite films are shown in Figure 12. The films had a thickness of $0.04 \pm 0.005 \mathrm{~mm}$ and were obtained by casting. The screw insertion temperatures were $160,175,175$, and $170{ }^{\circ} \mathrm{C}$. The WVP of the pure PLLA thin film was $4.688 \times 10^{-7} \mathrm{~g} \cdot \mathrm{m} / \mathrm{m}^{2} \cdot \mathrm{s} \cdot \mathrm{pa}$. The WVP decreased to $3.797 \times 10^{-7} \mathrm{~g} \cdot \mathrm{m} / \mathrm{m}^{2} \cdot \mathrm{s} \cdot \mathrm{pa}$ after adding $0.5 \%$ GO-g-PLLA, and this value is $19 \%$ lower than that of pure PLLA. Further decreases in WVP were not pronounced after adding $1 \%$ or $1.5 \%$ GO-g-PLLA, because with additional GO-g-PLLA, the large amount of hydrophilic oxygen-containing functional groups on the GO surface can easily adsorb water, thus increasing the composite absorption capacity of water vapor.

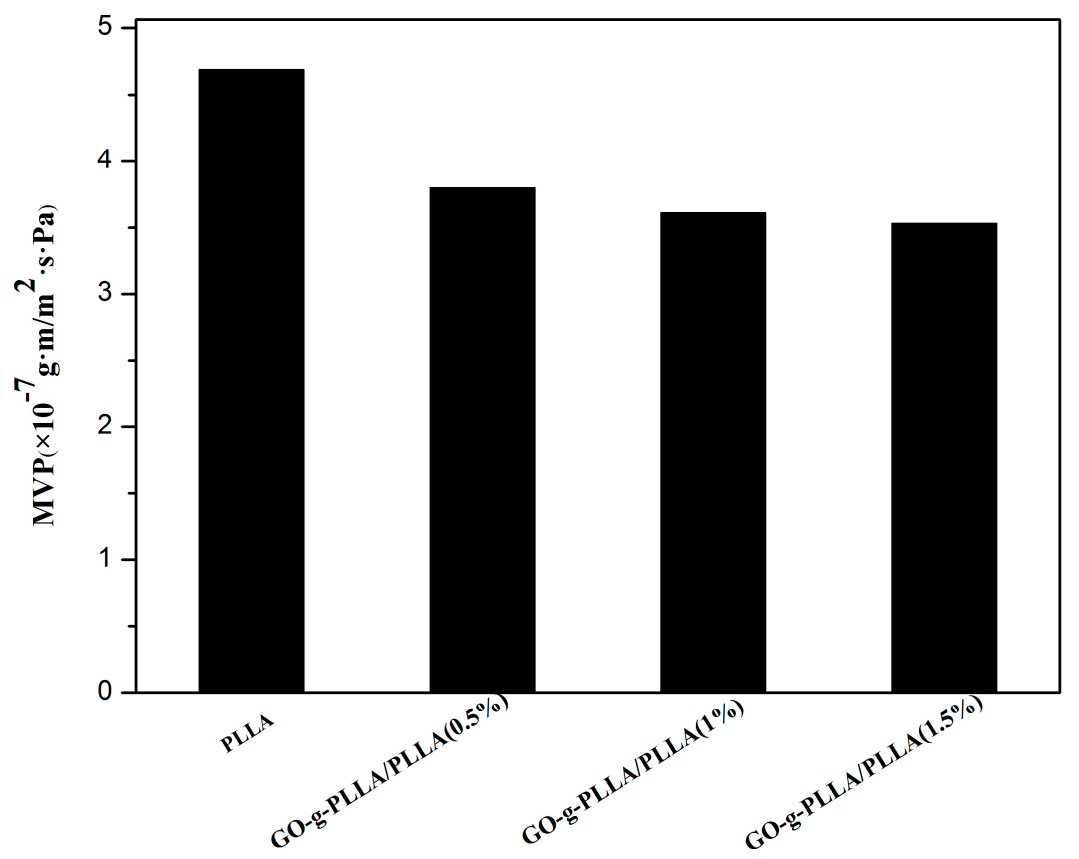

Figure 12. PLLA and GO-g-PLLA/PLLA Water Vapor Permeability (WVP) results. 


\subsubsection{Oxygen Permeability $\left(\mathrm{PO}_{2}\right)$ Test}

The permeability was calculated using Equation (1). The test results are shown in Figure 13. The $\mathrm{PO}_{2}$ of PLLA thin film was $3.013\left(\mathrm{~cm}^{3} /\left(24 \mathrm{~h} \times \mathrm{m}^{2}\right) \times(\mathrm{cm} /\right.$ bar $)$. The oxygen barrier capacity decreased to $2.135\left(\mathrm{~cm}^{3} /\left(24 \mathrm{~h} \times \mathrm{m}^{2}\right) \times(\mathrm{cm} /\right.$ bar $)$ when $0.5 \mathrm{wt} \%$ GO-g-PLLA was added, which is a $29.14 \%$ reduction. When $2 \mathrm{wt} \% \mathrm{GO}-g$-PLLA was added, the $\mathrm{PO}_{2}$ of the composite thin film decreased to $1.611\left(\mathrm{~cm}^{3} /\left(24 \mathrm{~h} \times \mathrm{m}^{2}\right) \times(\mathrm{cm} / \mathrm{bar})\right.$, a reduction of $46.53 \%$. In addition, when the amount of GO- $g$-PLLA exceeded $2 \%$, the decrease in $\mathrm{PO}_{2}$ was not pronounced.

$$
\text { Permeability }=\text { OTR } \times(\text { thickness } / \Delta \mathrm{P})=\left[\mathrm{cm}^{3} /\left(\mathrm{m}^{2} \times 24 \mathrm{~h}\right)\right] \times(\mathrm{cm} / \text { bar })
$$

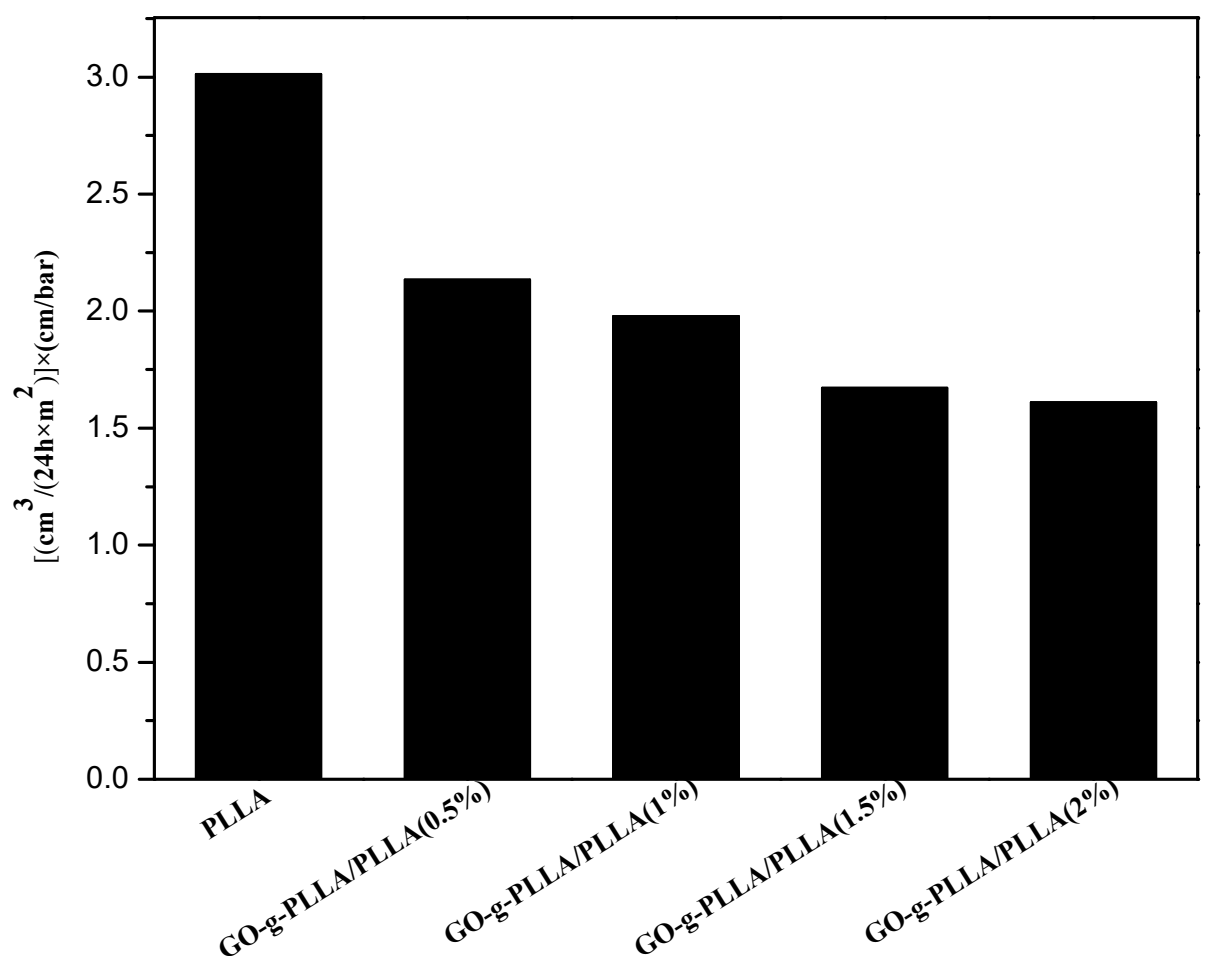

Figure 13. PLLA and GO-g-PLLA/PLLA oxygen permeability $\left(\mathrm{PO}_{2}\right)$ results.

\section{Conclusions}

We used hydroxyl groups on the surface of GO to initiate the ring-opening polymerization of lactic acid $O$-carboxyanhydride and successfully grafted PLLA onto the surface of GO surface to obtain the GO-g-PLLA composite. Compared to studies in the literature, this method is metal-free and under mild conditions $\left(25^{\circ} \mathrm{C}\right)$. For PLLA with graphene and GO composite materials, the literature focuses on the study of the crystallinity and heat resistance of PLLA. Our study shows that the functional materials prepared by this method largely improve the compatibility and the tensile and barrier properties of the resulting composite materials. When $0.5 \mathrm{wt} \%$ of GO-g-PLLA was added, the tensile strength and tensile fracture strength of the GO-g-PLLA/PLLA composite increased by $48.6 \%$ and $39 \%$, respectively, compared to those of PLLA, whereas the water vapor and oxygen permeabilities of the GO-g-PLLA/PLLA composite decreased by $19 \%$ and $29 \%$, respectively, compared to the neat polymer. Improving the gas barrier performance and flexibility of PLLA for the purpose of packaging and other uses will help to improve the range of its uses in practical applications. 
Acknowledgments: This work was supported by the National Natural Science Foundation of China (Project Nos. 31460247, 81460542, 81760644), the Biodegradable Materials Innovative Research Team (in Science and Technology) at the University of Yunnan Province and the Innovation Team Based on Research and Application of Biological Functional Materials of Yunnan Minzu University (2017HC034).

Author Contributions: This paper was accomplished based on the collaborative work of the authors. Mingwei Yuan performed the experiments, analyzed the data, interpreted the experimental results, and wrote the paper. Chengdong Xiong and Minglong Yuan supervised the entire research progress and contributed to the experimental design.

Conflicts of Interest: The authors declare no conflict of interest.

\section{References}

1. Rasal, R.M.; Janorkar, A.V.; Hirt, D.E. Poly(lactic acid) modifications. Prog. Polym. Sci. 2010, 35, $338-356$. [CrossRef]

2. Anderson, K.S.; Schreck, K.M.; Hillmyer, M.A. Toughening polylactide. Polym. Rev. 2008, 48, 85-108. [CrossRef]

3. Xu, J.-Z.; Chen, T.; Yang, C.-L.; Li, Z.-M.; Mao, Y.-M.; Zeng, B.-Q.; Hsiao, B.S. Isothermal crystallization of poly(L-lactide) induced by graphene nanosheets and carbon nanotubes: A comparative study. Macromolecules 2010, 43, 5000-5008. [CrossRef]

4. Murariu, M.; Dechief, A.L.; Bonnaud, L.; Paint, Y.; Gallos, A.; Fontaine, G.; Bourbigot, S.; Dubois, P. The production and properties of polylactide composites filled with expanded graphite. Polym. Degrad. Stab. 2010, 95, 889-900. [CrossRef]

5. Cao, Y.; Feng, J.; Wu, P. Preparation of organically dispersible graphene nanosheet powders through a lyophilization method and their poly(lactic acid) composites. Carbon 2010, 48, 3834-3839. [CrossRef]

6. Wang, H.; Qiu, Z. Crystallization kinetics and morphology of biodegradable poly(L-lactic acid)/graphene oxide nanocomposites: Influences of graphene oxide loading and crystallization temperature. Thermochim. Acta 2012, 527, 40-46. [CrossRef]

7. Wang, H.; Qiu, Z. Crystallization behaviors of biodegradable poly(L-lactic acid)/graphene oxide nanocomposites from the amorphous state. Thermochim. Acta 2011, 526, 229-236. [CrossRef]

8. Liu, S.; Gordiichuk, P.; Wu, Z.-S.; Liu, Z.; Wei, W.; Wagner, M.; Mohamed-Noriega, N.; Wu, D.; Mai, Y.; Herrmann, A.; et al. Patterning two-dimensional free-standing surfaces with mesoporous conducting polymers. Nat. Commun. 2015, 6, 8817. [CrossRef] [PubMed]

9. Liu, S.; Zhang, J.; Dong, R.; Gordiichuk, P.; Zhang, T.; Zhuang, X.; Mai, Y.; Liu, F.; Herrmann, A.; Feng, X. Two-dimensional mesoscale-ordered conducting polymers. Angew. Chem. Int. Ed. 2016, 55, 12516-12521. [CrossRef] [PubMed]

10. Sun, Y.; He, C. Synthesis and stereocomplex crystallization of poly(lactide)-graphene oxide nanocomposites. ACS Macro Lett. 2012, 1, 709-713. [CrossRef]

11. Arias, V.; Odelius, K.; Hoglund, A.; Albertsson, A.-C. Homocomposites of polylactide (PLLA) with induced interfacial stereocomplex crystallites. ACS Sustain. Chem. Eng. 2015, 3, 2220-2231. [CrossRef] [PubMed]

12. Xu, H.; Wu, D.; Yang, X.; Xie, L.; Hakkarainen, M. Thermostable and impermeable "nano-barrier walls" constructed by poly(lactic acid) stereocomplex crystal decorated graphene oxide nanosheets. Macromolecules 2015, 48, 2127-2137. [CrossRef]

13. Xu, H.; Xie, L.; Wu, D.; Hakkarainen, M. Immobilized graphene oxide nanosheets as thin but strong nanointerfaces in biocomposites. ACS Sustain. Chem. Eng. 2016, 4, 2211-2222. [CrossRef]

14. Xu, H.; Feng, Z.-X.; Xie, L.; Hakkarainen, M. Graphene oxide-driven design of strong and flexible biopolymer barrier films: From smart crystallization control to affordable engineering. ACS Sustain. Chem. Eng. 2016, 4, 334-349. [CrossRef]

15. Hua, L.; Kai, W.; Yang, J.; Inoue, Y. A new poly(L-lactide)-grafted graphite oxide composite: Facile synthesis, electrical properties and crystallization behaviors. Polym. Degrad. Stab. 2010, 95, 2619-2627. [CrossRef]

16. Kricheldorf, H.R.; Jonte, J.M. New polymer syntheses. 8. Synthesis and polymerization of L-lactic acid O-carboxyanhydride (5-methyl-dioxolan-2,4-dione). Polym. Bull. 1983, 9, 276-283.

17. Bonduelle, C.; Martin-Vaca, B.; Bourissou, D. Lipase-catalyzed ring-opening polymerization of the O-carboxylic anhydride derived from lactic acid. Biomacromolecules 2009, 10, 3069-3073. [CrossRef] [PubMed] 
18. Thillaye du Boullay, O.; Marchal, E.; Martin-Vaca, B.; Cossio, F.P.; Bourissou, D. An activated equivalent of lactide toward organocatalytic ring-opening polymerization. J. Am. Chem. Soc. 2006, 128, 16442-16443. [CrossRef] [PubMed]

19. Chen, C.; Chen, L.; Chen, H.; Li, H.; Yuan, M. Synthesis and solubility of polylactide-co-poly(amino acid) random copolymer. Polym. Bull. 2013, 70, 1739-1751. [CrossRef]

20. Yuan, M.L.; Li, X.H.; Xiong, C.D.; Deng, X.M. Polymerization of lactides and lactones-5. Ring-opening polymerization of epsilon-caprolactone and dl-lactide by rare earth 2-methylphenyl samarium. Eur. Polym. J. 1999, 35, 2131-2138. [CrossRef]

21. Deng, X.M.; Yuan, M.L.; Xiong, C.D.; Li, X.H. Polymerization of lactides and lactones. II. Ring-opening polymerization of epsilon-caprolactone and DL-lactide by organoacid rare earth compounds. J. Appl. Polym. Sci. 1999, 71, 1941-1948. [CrossRef]

22. Yuan, M.L.; Xiong, C.D.; Li, X.H.; Deng, X.M. Polymerization of lactides and lactones. III. Ring-opening polymerization of DL-lactide by the (eta(3)-c3h5)(2) $\mathrm{sm}(\mathrm{mu}(2)-\mathrm{cl})(2)(\mathrm{mu}(3)-\mathrm{cl}) \mathrm{mg}(\mathrm{tmed})(\mathrm{mu}(2)-\mathrm{cl}) \mathrm{mg}(\mathrm{tmed})$ complex. J. Appl. Polym. Sci. 1999, 73, 2857-2862. [CrossRef]

23. Deng, X.M.; Yuan, M.L.; Xiong, C.D.; Li, X.H. Polymerization of lactides and lactones. IV. Ring-opening polymerization of epsilon-caprolactone by rare earth phenyl compounds. J. Appl. Polym. Sci. 1999, 73, 1401-1408. [CrossRef]

24. Deng, X.M.; Yuan, M.L.; Li, X.H.; Xiong, C.D. Polymerization of lactides and lactones vii. Ring-opening polymerization of lactide by rare earth phenyl compounds. Eur. Polym. J. 2000, 36, 1151-1156. [CrossRef]

25. Yuan, M.L.; Wang, Y.H.; Li, X.H.; Xiong, C.D.; Deng, X.M. Polymerization of lactides and lactones. 10. Synthesis, characterization, and application of amino-terminated poly(ethylene glycol)-co-poly(epsilon-caprolactone) block copolymer. Macromolecules 2000, 33, 1613-1617. [CrossRef]

26. Fang, M.; Wang, K.; Lu, H.; Yang, Y.; Nutt, S. Covalent polymer functionalization of graphene nanosheets and mechanical properties of composites. J. Mater. Chem. 2009, 19, 7098-7105. [CrossRef]

27. Wang, J.; Shi, Z.; Ge, Y.; Wang, Y.; Fan, J.; Yin, J. Functionalization of unzipped carbon nanotube via in situ polymerization for mechanical reinforcement of polymer. J. Mater. Chem. 2012, 22, 17663-17670. [CrossRef]

28. Song, W.; Zheng, Z.; Tang, W.; Wang, X. A facile approach to covalently functionalized carbon nanotubes with biocompatible polymer. Polymer 2007, 48, 3658-3663. [CrossRef]

29. Yoon, J.T.; Lee, S.C.; Jeong, Y.G. Effects of grafted chain length on mechanical and electrical properties of nanocomposites containing polylactide-grafted carbon nanotubes. Compos. Sci. Technol. 2010, 70, 776-782. [CrossRef]

30. Goffin, A.-L.; Raquez, J.-M.; Duquesne, E.; Siqueira, G.; Habibi, Y.; Dufresne, A.; Dubois, P. From interfacial ring-opening polymerization to melt processing of cellulose nanowhisker-filled polylactide-based nanocomposites. Biomacromolecules 2011, 12, 2456-2465. [CrossRef] [PubMed]

31. Yoon, J.T.; Jeong, Y.G.; Lee, S.C.; Min, B.G. Influences of poly(lactic acid)-grafted carbon nanotube on thermal, mechanical, and electrical properties of poly(lactic acid). Polym. Adv. Technol. 2009, 20, 631-638. [CrossRef]

32. Amirian, M.; Chakoli, A.N.; Sui, J.H.; Cai, W. Enhanced mechanical and photoluminescence effect of poly(L-lactide) reinforced with functionalized multiwalled carbon nanotubes. Polym. Bull. 2012, 68, 1747-1763. [CrossRef]

33. Olalde, B.; Aizpurua, J.M.; Garcia, A.; Bustero, I.; Obieta, I.; Jurado, M.J. Single-walled carbon nanotubes and multiwalled carbon nanotubes functionalized with poly(L-lactic acid): A comparative study. J. Phys. Chem. C 2008, 112, 10663-10667. [CrossRef]

34. Chen, G.X.; Kim, H.S.; Park, B.H.; Yoon, J.S. Controlled functionalization of multiwalled carbon nanotubes with various molecular-weight poly(L-lactic acid). J. Phys. Chem. B 2005, 109, 22237-22243. [CrossRef] [PubMed]

35. Chen, L.; Xu, Z.; Li, J.; Li, Y.; Shan, M.; Wang, C.; Wang, Z.; Guo, Q.; Liu, L.; Chen, G.; et al. A facile strategy to prepare functionalized graphene via intercalation, grafting and self-exfoliation of graphite oxide. J. Mater. Chem. 2012, 22, 13460-13463. [CrossRef]

36. Yang, J.-H.; Lin, S.-H.; Lee, Y.-D. Preparation and characterization of poly(L-lactide)-graphene composites using the in situ ring-opening polymerization of PLLA with graphene as the initiator. J. Mater. Chem. 2012, 22, 10805-10815. [CrossRef] 
37. Li, W.; Xu, Z.; Chen, L.; Shan, M.; Tian, X.; Yang, C.; Lv, H.; Qian, X. A facile method to produce graphene oxide-g-poly(L-lactic acid) as an promising reinforcement for plla nanocomposites. Chem. Eng. J. 2014, 237, 291-299. [CrossRef]

38. Stankovich, S.; Dikin, D.A.; Piner, R.D.; Kohlhaas, K.A.; Kleinhammes, A.; Jia, Y.; Wu, Y.; Nguyen, S.T.; Ruoff, R.S. Synthesis of graphene-based nanosheets via chemical reduction of exfoliated graphite oxide. Carbon 2007, 45, 1558-1565. [CrossRef]

39. Dreyer, D.R.; Park, S.; Bielawski, C.W.; Ruoff, R.S. The chemistry of graphene oxide. Chem. Soc. Rev. 2010, 39, 228-240. [CrossRef] [PubMed]

40. Sun, Y.; He, C. Synthesis, stereocomplex crystallization, morphology and mechanical property of poly(lactide)-carbon nanotube nanocomposites. RSC Adv. 2013, 3, 2219-2226. [CrossRef]

41. Shen, J.; Hu, Y.; Shi, M.; Lu, X.; Qin, C.; Li, C.; Ye, M. Fast and facile preparation of graphene oxide and reduced graphene oxide nanoPLLAtelets. Chem. Mater. 2009, 21, 3514-3520. [CrossRef]

42. Cao, A.; Liu, Z.; Chu, S.; Wu, M.; Ye, Z.; Cai, Z.; Chang, Y.; Wang, S.; Gong, Q.; Liu, Y. A facile one-step method to produce graphene-cds quantum dot nanocomposites as promising optoelectronic materials. Adv. Mater. 2010, 22, 103-106. [CrossRef] [PubMed]

43. He, L.; Sun, J.; Wang, X.; Fan, X.; Zhao, Q.; Cai, L.; Song, R.; Ma, Z.; Huang, W. Unzipped multiwalled carbon nanotubes-incorporated poly(L-lactide) nanocomposites with enhanced interface and hydrolytic degradation. Mater. Chem. Phys. 2012, 134, 1059-1066. [CrossRef]

44. Leszczynska, A.; Pielichowski, K. Application of thermal analysis methods for characterization of polymer/montmorillonite nanocomposites. J. Therm. Anal. Calorim. 2008, 93, 677-687. [CrossRef]

45. Suk, J.W.; Piner, R.D.; An, J.; Ruoff, R.S. Mechanical properties of mono layer graphene oxide. ACS Nano 2010, 4, 6557-6564. [CrossRef] [PubMed]

46. Ramanathan, T.; Abdala, A.A.; Stankovich, S.; Dikin, D.A.; Herrera-Alonso, M.; Piner, R.D.; Adamson, D.H.; Schniepp, H.C.; Chen, X.; Ruoff, R.S.; et al. Functionalized graphene sheets for polymer nanocomposites. Nat. Nanotechnol. 2008, 3, 327-331. [CrossRef] [PubMed]

47. Sengupta, R.; Bhattacharya, M.; Bandyopadhyay, S.; Bhowmick, A.K. A review on the mechanical and electrical properties of graphite and modified graphite reinforced polymer composites. Prog. Polym. Sci. 2011, 36, 638-670. [CrossRef]

(c) 2018 by the authors. Licensee MDPI, Basel, Switzerland. This article is an open access article distributed under the terms and conditions of the Creative Commons Attribution (CC BY) license (http:/ / creativecommons.org/licenses/by/4.0/). 